\title{
A New Multi-Objective Evolutionary Approach to Graph Coloring and Channel Allocation Problems
}

\author{
S. Balakrishnan ${ }^{1}$, Tamilarasi Suresh ${ }^{2}$, Raja Marappan ${ }^{3, *}$ \\ ${ }^{1}$ Dr MGR Educational and Research Institute, Maduravoyal, Chennai, India. \\ ${ }^{2}$ Department of Information Technology, St. Peter's Institute of Higher Education and Research, Avadi, Chennai, India. \\ ${ }^{3}$ School of Computing, SASTRA Deemed University, Thanjavur, Tamil Nadu, India.
}

How to cite this paper: S. Balakrishnan, Tamilarasi Suresh, Raja Marappan. (2021) A New Multi-Objective Evolutionary Approach to Graph Coloring and Channel Allocation Problems. Journal of Applied Mathematics and Computation, 5(4), 252-263.

DOI: $10.26855 /$ jamc.2021.12.003

Received: September 6, 2021

Accepted: September 30, 2021

Published: October 14, 2021

*Corresponding author: Raja Marappan, School of Computing, SASTRA Deemed University, Thanjavur, Tamil Nadu, India.

Email: raja_csmath@cse.sastra.edu

\begin{abstract}
Recent years, a large amount of graph coloring algorithms have been applied in different disciplines and engineering. This paper exhibits a new multi-objective evolutionary algorithm using the new evolutionary operators with multi-objectives in finding the solution to graph coloring and channel allocation. Clique, a maximal connected complete subgraph of a given graph is obtained in solving channel allocation problem. The outcomes of the devised evolutionary method are compared with other recent approaches. Multiple better gene sequences are selected and are applying the subsequent crossover and mutation operations with multiple objectives. The devised operators significantly minimize the problem search space and average generations compared to the standard genetic algorithm. The proposed operators achieve better solution compared to some of the existing well known methods. The expected performance measures are also increased while minimizing the expected generations. The proposed operators can also be applied in solving special engineering applications of graph coloring.
\end{abstract}

\section{Keywords}

Evolutionary Algorithm, Graph Coloring, Channel Allocation, NP-hard

\section{Introduction}

A simple graph $G=(V, E)$ consists of a vertex set $V(G)=\left\{v_{1}, v_{2}, \ldots, v_{n}\right\}$ with $n$ vertices and edge set $E(G)=\left\{e_{1}, e_{2}, \ldots\right.$, $\left.e_{m}\right\}$ with $m$ edges such that each edge $e_{i} \in E(G)$ is uniquely incident with a vertex pair $\left(v_{j}, v_{k}\right) \in E(G), 1 \leq i \leq m, 1 \leq j, k \leq$ $n$ [1]. $A(G)$ is an $n x n$ adjacency matrix of $G$ such that $A_{j k}$ assigns 1 if there exists an edge joining vertices $v_{k}$ and $v_{j}$; otherwise, $A_{j k}$ assigns 0 [2]. Graph coloring is an assignment of color values to vertices of a $G$ subject to certain constraints. It assigns integers to $\mathrm{V}(\mathrm{G})$ such that no two adjacent vertices ar are assigned the same integer. Graph coloring is applied in different problems [34-37]. Graph Coloring Problem (GCP) is finding the value of a chromatic number, $\chi(G)$ that is the least number of integers required to assign $V(G)$ [1-3]. Different approximation and evolutionary methods are designed in solving GCP.

GCP is applied in different engineering applications. GCP can also be specifically applied in wireless networks where the cells are properly assigned to the corresponding channels based on its interference matrix called Channel Assignment Problem (CAP) [29, 38-41]. CAP allocates bandwidth and communication channels to base stations in cellular and wireless networks. The goal of CAP is to achieve maximum system spectral efficiency through channel reuse by avoiding the constraints of interference. The important criterion for next generation wireless systems is efficient spectrum utilization [53-54]. CAP defines the constraints: co-channel, adjacent channel and co-site constraints. Clique partitioning is applied in solving channel allocation schemes. Given the undirected graph $\mathrm{G}$, its clique $\mathrm{C}$ is a subgraph which consists of the 
vertex set $V^{\prime}$ in $V(G)$ such that there is an edge between every pair of vertices in V'. That is, C is the complete subgraph of $\mathrm{G}$. The split of $\mathrm{G}$ into a finite number of maximum cliques such that each vertex belongs to exactly a single clique is called clique partition, an NP-complete problem. Some of the other methods in solving graph coloring and its application are Particle Swarm Optimization (PSO) [18-19, 25-26], Ant Colony Optimization (ACO) [24], Cuckoo search [20], evolutionary, approximation, backtracking, branch and cut, column generation and heuristic search [4-11, 13-17, 21-23]. These methods are having certain limitations and are listed in Table 1 [42-50].

Table 1. Limitations in some well known methods

\begin{tabular}{|c|c|}
\hline Method & Limitations \\
\hline Branch and cut & Selection of upper bound is required \\
\hline Ant Colony Optimization & Recursive algorithm with more stack space is required \\
\hline Column Generation & Computationally complex operations are performed \\
\hline Semi-definite Programming & Near optimal solution is obtained for some graphs \\
\hline Particle Swarm Optimization & More generations are required and applied for planar graphs \\
\hline DSATUR & Near optimal solution \\
\hline FROGSIM, FINOCCHI & Near optimal solution \\
\hline Genetic operators & Near optimal solution \\
\hline Evolutionary methods & More search space and expensive \\
\hline
\end{tabular}

GAs are heuristic and adaptive search methods which are based on "survival of fittest”. Recently genetic algorithms are applied in discovering communities in complex social networks and solving constraint optimization problems based upon the principles of natural evolution [55-56]. The proper design of new genetic operators can reduce the exploration of problem search space and expected generations. Fast stochastic convergence is obtained when minimizing the problem search space and reducing the expected generations. Hence this paper presents a new genetic method in solving GCP and CAP with clique partition strategy. New crossover and mutation operators with multiple objectives are introduced in this paper. These operators minimize the expected generations and also reduce the exploration of search space. These operators are also minimizing the minimum color for certain graphs compared to existing methods.

Section 2 formulates the mathematical model of CAP. The new genetic method with clique partitioning strategy is presented in Section 3. Section 4 analyses the theoretical results and stochastic convergence of the devised operators. The well known problems are experimented and its outcomes are compared with other methods and are presented in Section 5. Section 6 presents the conclusions.

\section{Mathematical Formulation of CAP}

In general, the wireless networks consist of $m$ channels and $n$ cells. The solution to CAP is defined in the vector space $\mathrm{F}$ which represents a symmetric binary matrix of size $\mathrm{n} \times \mathrm{m}$.

For each value of $\mathrm{j}$ and $\mathrm{k}(1 \leq \mathrm{j} \leq \mathrm{n}$ and $1 \leq \mathrm{k} \leq \mathrm{m})$,

$\mathrm{F}(\mathrm{j}, \mathrm{k})=1$ if cell $\mathrm{j}$ is assigned the kth channel; $\mathrm{F}(\mathrm{j}, \mathrm{k})=0$ otherwise.

\subsection{Demand Constraint}

A total of $d_{i}$ channels are required for cell $i$. This implies that the total number of ones in row $i$ of $F$ must be $d_{i}$. That is if the assignment to cell $i$ violates the demand constraint, then

$$
\sum_{q=1}^{m} F_{i q}-d_{i} \neq 0
$$

\subsection{Co-Site channel Constraint (CSC)}

If channel $p$ is within distance $c_{i i}$ from an already assigned channel q in cell (i.e., $|p-q|<\mathrm{c}_{i i}$ ), then channel $p$ must not be assigned to cell $i$. That is if the assignment of channel $p$ to cell $i$ violates CSC, then 


$$
\sum_{\substack{q=p-\left(c_{i i}-1\right) \\ q \neq p \\ 1 \leq q \leq m}}^{p+\left(c_{i i}-1\right)} F_{i q}>0
$$

\subsection{Co-Channel Constraint (CCC) \& Adjacent Channel Constraint (ACC)}

CCC requires that $\mathrm{c}_{\mathrm{ij}}=1$, that is, cells $\mathrm{i}$ and $\mathrm{j}$ must use channels that maintain a minimum separation of 1 unit. ACC requires that $\mathrm{c}_{\mathrm{ij}} \geq 2$, that is, cells $\mathrm{i}$ and $\mathrm{j}$ must use channels separated by at least 2 units. Hence for CCC \& ACC, if channel $p$ in cell $i$ is within distance from an already assigned channel $q$ in cell $j$, where $c_{i j}>0$ and $i \neq j$ (i.e., $|p-q|<c_{i j}$ ), then channel $p$ must not be assigned to cell $i$. That is, if the assignment of channel $p$ to cell $i$ violates CCC and/or ACC, then

$$
\sum_{\substack{j=1 \\ j \neq i}}^{n} \sum_{\substack{q=p-\left(c_{i j}-1\right) \\ c_{i j}>0}}^{p+\left(c_{i j}-1\right)} F_{j q}>0
$$

Hence the objective function of CAP is derived in the following cost function:

$$
C(F)=\sum_{i=1}^{n}\left(\sum_{q=1}^{m} F_{i q}-d_{i}\right)+\sum_{i=1}^{n} \sum_{p=1}^{m}\left(\sum_{\substack{q+\left(c_{i i}-1\right) \\ q \neq p \\ 1 \leq q \leq m}}^{p+\left(c_{i i}-1\right)} F_{i q}\right) F_{i p}+\sum_{i=1}^{n} \sum_{p=1}^{m}\left(\sum_{\substack{j=1 \\ j \neq i \\ c_{i j}>0}}^{n} \sum_{\substack{q=p-\left(c_{i j}-1\right) \\ 1 \leq q \leq m}}^{p+\left(c_{i j}-1\right)} F_{j q}\right) F_{i p}
$$

The objective function of CAP is to minimize $\mathrm{C}(\mathrm{F})$; that is, find an $\mathrm{F}$ such that $\mathrm{C}(\mathrm{F})$ reaches 0 .

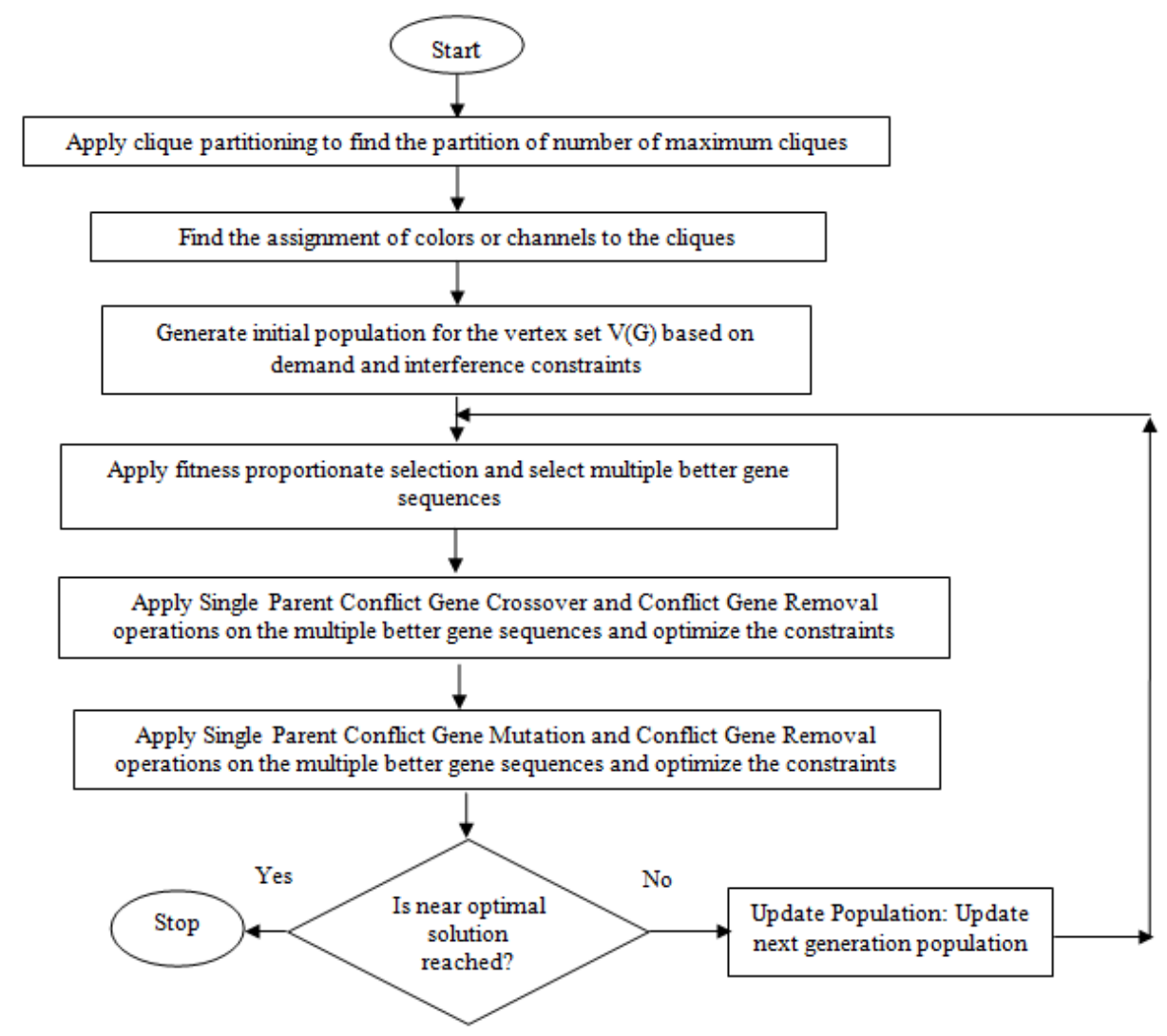

Figure 1. Flowchart of the proposed method in solving GCP \& CAP. 


\section{Proposed Genetic Algorithm}

The proposed method initially finds the number of maximum cliques for the given graph G. Then independent cliques are identified and solved based on the constraints. Starting with random initial population, fitness proportionate selection select multiple better gene sequences and are applied for crossover operation in the next stage. The new conflict based crossover and mutation operators are applied on more than one better individual with multiple objectives to produce the better offspring. This iterative procedure is continued until reaching the better solution.

The flowchart of this new method is presented in Figure 1. The clique partition strategy splits V(G) into different cliques. The strategy splits $G$ into a required number of maximum cliques to minimize the network interference in solving CAP. The independent cliques are identified and are colored or assigned the channels independently.

The general procedure to find the solution of CAP is given below:

a. Partition $V(G)$ into number of maximum cliques using clique partition;

b. Apply the proposed operators for the identified cliques;

c. Find the independent cliques and assign valid channels based on interference constraints;

G': (S, E') is constructed from $G$ using clique partition. Every vertex $s_{i}$ in $S$ consists of a set of finite vertices $v_{i}$ in $V$. A vertex $s_{i}$ in $S$ is a common neighbor of $s_{j}$ and $s_{k}$ in $S$ if there exists edges (i, j) and (i, k) in E'. The maximum clique is obtained in the vertex set $\mathrm{s}_{\mathrm{i}}$ in $\mathrm{S}$ using the following steps:

a. Put each $v_{i}$ in $V(G)$ in a distinct vertex $s_{i}$ in $S$ of $G^{\prime}$.

b. Identify the vertices $s_{i 1}, s_{i 2}$ in $S$ such that $\left(s_{i 1}, s_{i 2}\right)$ in $E^{\prime}(G)$ with large common adjacency.

c. Merge the vertices into a single vertex $s_{i 1 i 2}$ which have $s_{i 1}$ and $s_{i 2}$ in all.

d. Determine the intersection set which consists of the entire intersection neighbors of $s_{i 1}$ and $s_{i 2}$.

e. Remove all edges which are emerging from $s_{i 1}$ or $s_{i 2}$ in $G$ '.

f. $\quad$ Update new links from $s_{i 1 i 2}$ to all vertices.

g. Perform the operations (a) to ( $f$ ) repeatedly until $G$ has 0 edges.

Procedure Clique Partition

// construct $G^{\prime}\left(S, E^{\prime}\right)$

Initialize $S=\varphi$ and $E^{\prime}=\varphi$;

For all $v_{i} \in V(G)$ do $s_{i}=\left\{v_{i}\right\} ; S=S U\left\{s_{i}\right\}$;

For all $e_{i, j} \in E(G)$ do $E^{\prime}=E^{\prime} U\left\{e^{\prime}{ }_{i, j}\right\}$;

Repeat when $E^{\prime} \neq \varphi$ :

$m c=-1$;

For all $e_{i, j} \in E^{\prime}$ do

$c_{i, j}=$ cardinality of common neighbor $\left(G^{\prime}, s_{i}, s_{j}\right)$;

if $c_{i, j}>m c$

$m c=c_{i, j}$

intersection set $=$ intersection $\left(G^{\prime}, s_{\text {index } 1}, s_{\text {index } 2}\right)$;

$E^{\prime}=$ remove $\left(E^{\prime}, s_{\text {index } 1}\right)$;

$E^{\prime}=$ remove $\left(E^{\prime}, s_{\text {index } 2}\right)$;

$S_{\text {index1index2 } 2}=S_{\text {index } 1} U S_{\text {index } 2 \text {; }}$

$S=S-S_{\text {index } 1}-S_{\text {index } 2}$;

$S=S U\left\{S_{\text {index } 1 \text { index } 2}\right\}$

Update E'for all $s_{i} \epsilon$ intersection set

The new genetic operators are designed as follows:

\subsection{Crossover Operation}

The following are the steps in new recombination operator.

a. Apply the following steps for the selected gene sequences.

b. Choose $p_{\text {cr. }}$

c. If $p_{c r}>p_{c}$ then apply the conflict color recombination and update offspring.

d. Apply the removal of conflict colors for fixed times.

e. Repeat this operation for fixed iterations to remove the conflicts.

f. Apply crossover at single, multiple points on the offspring i' and j' to produce new offspring by optimizing the constraints.

g. Repeat the above steps for finite iterations. 


\subsection{Mutation Operation}

The mutation is performed when $p_{m}<p_{m r}$ fined as follows:

a. Identify the conflicting individuals.

b. Update new individuals with minimum change in gene values.

c. Perform the gene updates to remove the total conflicts.

d. Apply mutation at single, multiple points and update the better offspring by optimizing the constraints.

e. $\quad$ Repeat the above steps for finite iterations.

\section{Analysis and Stochastic Convergence}

The new genetic operators are obtained better solution and achieve the stochastic convergence after reaching a fixed value of $g$. The proposed evolutionary operators achieve stochastic convergence in getting better near optimal solution.

Proof for the work carried to achieve stochastic convergence: Let $\mathrm{S}$ represents the finite search space. For arbitrarily chosen individuals $\mathrm{i}, \mathrm{j} \in \mathrm{S}$, individuals should satisfy reachability condition.

Let $\mathrm{p}_{\mathrm{c}}$ and $\mathrm{p}_{\mathrm{m}}$ define the crossover and mutation probabilities for applying the proposed crossover and mutation.

Then the probability of generating i" is given by

Probability (i") $\geq$

Probability of crossover x $\mathrm{p}\left\{\mathrm{i}^{\prime}=\right.$ crossover (i) $\}$ x Probability of mutation $\mathrm{x}\left\{\mathrm{i}^{\prime \prime}=\right.$ mutation (i') $\}$

Clearly $\mathrm{p}\left\{\mathrm{i}^{\prime}=\right.$ recombination $\left.(\mathrm{i})\right\}>0$ and $\mathrm{p}\left\{\mathrm{i}^{\prime \prime}=\operatorname{mutation}\left(\mathrm{i}^{\prime}\right)\right\}>0$.

Thus, the probability of generating i" lies in 0 and 1 and hence i reaches to i" [12].

The elitism operation replaces the worst individual with better one and $P_{g}$ is updated. The devised operators reduce $F_{g}(j)$ $\& \mathrm{~F}_{\mathrm{g}}(\mathrm{i})$ during the generations and the following inferences are obtained:

Inference 1: Either $F_{g}(i)$ or $F_{g}(j)$ decreases to obtain the near solution.

$$
\begin{aligned}
& F_{0}(i) \geq F_{1}(i) \geq F_{2}(i) \geq \ldots \geq F_{q}(i) \geq F_{q+1}(i)=0 . \\
& F_{0}(j) \geq F_{1}(j) \geq F_{2}(j) \geq \ldots \geq F_{q}(j) \geq F_{q+1}(j)=0 .
\end{aligned}
$$

Inference 2: $F_{g}(i)$ or $F_{g}(j)$ satisfies the following relationship for finite generations:

$$
\begin{aligned}
& F_{0}(i) \geq F_{1}(i) \geq F_{2}(i) \geq \ldots \geq F_{q}(i) \\
& F_{0}(j) \geq F_{1}(j) \geq F_{2}(j) \geq \ldots \geq F_{q}(j)
\end{aligned}
$$

Then after finite generations the following relationship achieves the near optimal solution.

$$
\begin{aligned}
& \mathrm{F}_{\mathrm{q}}(\mathrm{i})<\mathrm{F}_{\mathrm{q}+1}(\mathrm{i})<\ldots<\mathrm{F}_{\mathrm{t}}(\mathrm{i}) \\
& \mathrm{F}_{\mathrm{q}}(\mathrm{j})<\mathrm{F}_{\mathrm{q}+1}(\mathrm{j})<\ldots<\mathrm{F}_{\mathrm{t}}(\mathrm{j}) .
\end{aligned}
$$

Thus the proposed operators prove the stochastic convergence in obtaining better near optimal solution.

\section{Results and Analysis}

The proposed method is experimented in solving some of the benchmark graphs and channel allocation problems using Intel Core i5-2450M 2.5GHz system using Java [27]. The graph instances $\mathrm{n}$ and $\mathrm{m}$ are used in evaluating the performance measurements of the method. The outcomes are shown in Tables 2, 3 and 4 with the following inferences:

1. Stochastic convergence is reached even to the high density graphs, such as miles 1500 .col.

2. The expected generations $\bar{g}$ is reduced compared to other genetic methods and the obtained values are shown in figures from Figure 2 to Figure 4.

3. Solution is obtained with minimal complexity for queen, register allocation, Miles graphs.

4. Better near solution is obtained for latin_square_10.col.

When comparing the results of this method with some of the existing methods, this new method offsets the problems such as improving solution, search space reduction, and achieve quick convergence for minimal N [25-58].

The important findings are as follows:

1. Solution is obtained for different families of random graphs.

2. Stochastic convergence is achieved for small $\mathrm{N}<10$ compared to other methods.

3. Solution is obtained for queen, Mycielski graphs compared to near-optimal performance of existing methods. 
4. Provides better solution than DSATUR, FINOCCHI and FROGSIM methods.

The comparison of results obtained with the other strategies is shown in Table 5. Table 6 represents the different CAP instances and the expected generations obtained are given in Figure 5. The convergence of near optimal solution is shown in Table 7. This new method significantly reduces the expected generations and achieves higher frequency of convergence for the benchmark and random instances.

Table 2. Computation of Near Optimal Solution on Small Graphs $(\mathbf{n}<\mathbf{1 0 0})$

\begin{tabular}{ccccccc}
\hline \multirow{2}{*}{ Graph No } & & Graph $(\mathrm{G})$ & & \multicolumn{2}{c}{ Minimum color obtained in } \\
Proposed method
\end{tabular}

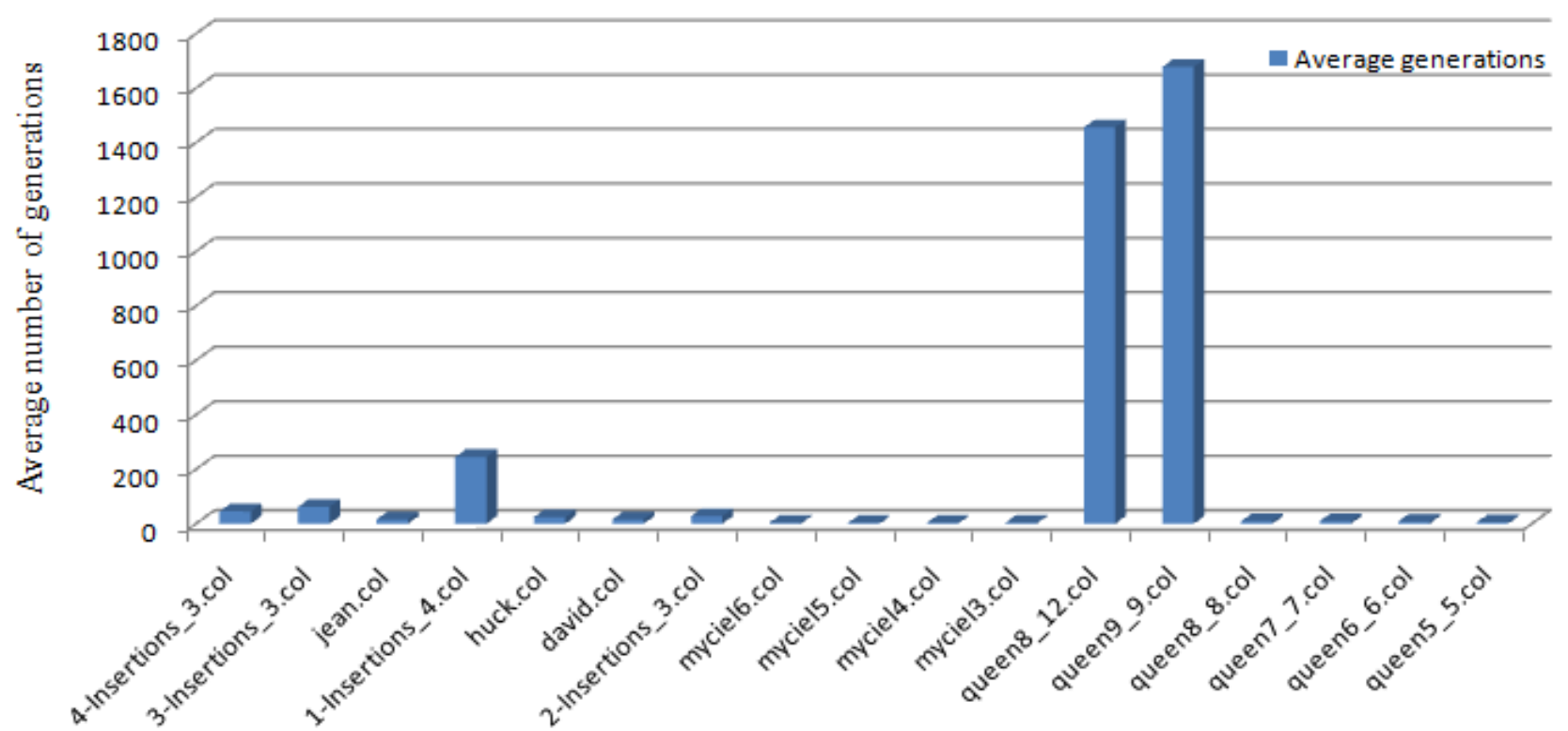

Small graphs

Figure 2. Expected generations obtained for small graphs. 


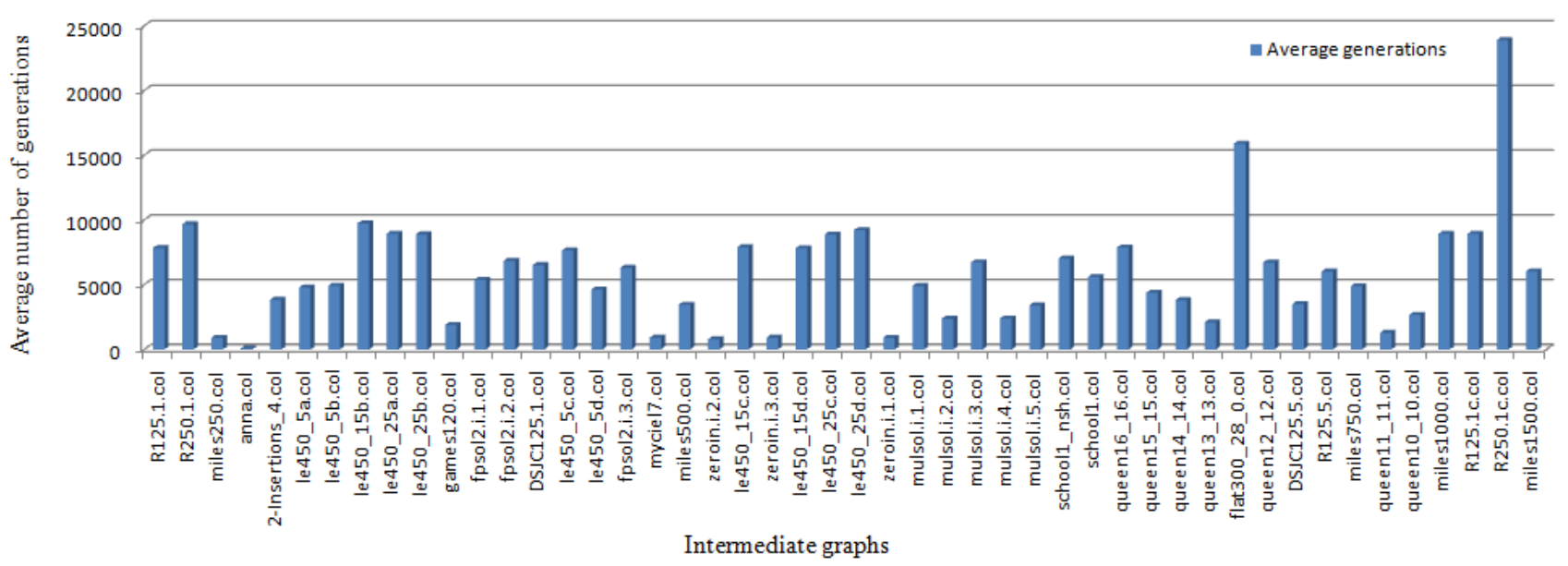

Figure 3. Expected generations obtained for intermediate graphs.

Table 3. Computation of Near Optimal Solution on Intermediate Graphs $(\mathbf{1 0 0} \leq \mathbf{n}<500)$

\begin{tabular}{|c|c|c|c|c|c|c|}
\hline \multirow{2}{*}{ Graph No. } & \multicolumn{4}{|c|}{ Graph (G) } & \multicolumn{2}{|c|}{ Minimum color obtained in } \\
\hline & Graph Type & Instances & $\chi(G)$ & Density & Existing method & Proposed method \\
\hline 18 & myciel7.col & $\mathrm{n}=191 ; \mathrm{m}=2360$ & 8 & 0.13 & 8 & 8 \\
\hline 19 & games120.col & $\mathrm{n}=120 ; \mathrm{m}=638$ & 9 & 0.09 & 9 & 9 \\
\hline 20 & miles250.col & $\mathrm{n}=128 ; m=387$ & 8 & 0.05 & 8 & 8 \\
\hline 21 & anna.col & $\mathrm{n}=138 ; \mathrm{m}=493$ & 11 & 0.05 & 11 & 11 \\
\hline 22 & queen10_10.col & $\mathrm{n}=100 ; \mathrm{m}=2940$ & 11 & 0.59 & 11 & 11 \\
\hline 23 & queen12_12.col & $n=144 ; m=5192$ & 12 & 0.50 & 12 & 12 \\
\hline 24 & queen14_14.col & $\mathrm{n}=196 ; \mathrm{m}=8372$ & 14 & 0.44 & 14 & 14 \\
\hline 25 & queen15_15.col & $\mathrm{n}=225 ; \mathrm{m}=10360$ & 15 & 0.41 & 15 & 15 \\
\hline 26 & queen16_16.col & $\mathrm{n}=256 ; \mathrm{m}=12640$ & 16 & 0.39 & 16 & 16 \\
\hline 27 & queen11_11.col & $\mathrm{n}=121 ; \mathrm{m}=3960$ & 11 & 0.55 & 11 & 11 \\
\hline 28 & queen13_13.col & $n=169 ; m=6656$ & 13 & 0.47 & 13 & 13 \\
\hline 29 & miles500.col & $\mathrm{n}=128 ; m=1170$ & 20 & 0.14 & 20 & 20 \\
\hline 30 & miles750.col & $n=128 ; m=4226$ & 31 & 0.52 & 31 & 31 \\
\hline 31 & miles1000.col & $\mathrm{n}=128 ; \mathrm{m}=6432$ & 42 & 0.79 & 42 & 42 \\
\hline 32 & miles1500.col & $\mathrm{n}=128 ; m=10396$ & 73 & 1.28 & 73 & 73 \\
\hline 33 & zeroin.i.1.col & $\mathrm{n}=211 ; \mathrm{m}=4100$ & 49 & 0.19 & 49 & 49 \\
\hline 34 & zeroin.i.2.col & $\mathrm{n}=211 ; \mathrm{m}=3541$ & 30 & 0.16 & 30 & 30 \\
\hline 35 & zeroin.i.3.col & $\mathrm{n}=206 ; \mathrm{m}=3540$ & 30 & 0.17 & 30 & 30 \\
\hline 36 & mulsol.i.1.col & $n=197 ; m=3925$ & 49 & 0.20 & 49 & 49 \\
\hline 37 & mulsol.i.2.col & $\mathrm{n}=188 ; m=3885$ & 31 & 0.22 & 31 & 31 \\
\hline 38 & mulsol.i.3.col & $n=184 ; m=3916$ & 31 & 0.23 & 31 & 31 \\
\hline 39 & mulsol.i.4.col & $\mathrm{n}=185 ; \mathrm{m}=3946$ & 31 & 0.23 & 31 & 31 \\
\hline 40 & mulsol.i.5.col & $n=186 ; m=3973$ & 31 & 0.23 & 31 & 31 \\
\hline 41 & le450_5a.col & $\mathrm{n}=450 ; \mathrm{m}=5714$ & 5 & 0.06 & 5 & 5 \\
\hline 42 & le450_5b.col & $\mathrm{n}=450 ; \mathrm{m}=5734$ & 5 & 0.06 & 5 & 5 \\
\hline 43 & le450_5c.col & $n=450 ; m=9803$ & 5 & 0.10 & 5 & 5 \\
\hline 44 & le450_5d.col & $\mathrm{n}=450 ; \mathrm{m}=9757$ & 5 & 0.10 & 5 & 5 \\
\hline 45 & le450_15b.col & $\mathrm{n}=450 ; m=8169$ & 15 & 0.08 & 15 & 15 \\
\hline 46 & le450_15c.col & $\mathrm{n}=450 ; m=16680$ & 15 & 0.16 & 15 & 15 \\
\hline 47 & le450_15d.col & $\mathrm{n}=450 ; \mathrm{m}=16750$ & 15 & 0.17 & 15 & 15 \\
\hline 48 & le450_25a.col & $\mathrm{n}=450 ; \mathrm{m}=8260$ & 25 & 0.08 & 25 & 25 \\
\hline 49 & le450_25b.col & $n=450 ; m=8263$ & 25 & 0.08 & 25 & 25 \\
\hline
\end{tabular}




\begin{tabular}{|c|c|c|c|c|c|c|}
\hline 50 & le450_25c.col & $\mathrm{n}=450 ; \mathrm{m}=17343$ & 25 & 0.17 & 26 & 25 \\
\hline 51 & le450_25d.col & $\mathrm{n}=450 ; \mathrm{m}=17425$ & 25 & 0.17 & 26 & 25 \\
\hline 52 & school1.col & $\mathrm{n}=385 ; \mathrm{m}=19095$ & 14 & 0.26 & 14 & 14 \\
\hline 53 & school1_nsh.col & $\mathrm{n}=352 ; \mathrm{m}=14612$ & 14 & 0.24 & 14 & 14 \\
\hline 54 & fpsol2.i.1.col & $n=496 ; m=11654$ & 65 & 0.09 & 65 & 65 \\
\hline 55 & fpsol2.i.2.col & $\mathrm{n}=451 ; \mathrm{m}=8691$ & 30 & 0.09 & 30 & 30 \\
\hline 56 & fpsol2.i.3.col & $n=425 ; m=8688$ & 30 & 0.10 & 30 & 30 \\
\hline 57 & 2-Insertions_4.col & $\mathrm{n}=149 ; \mathrm{m}=541$ & 4 & 0.05 & 4 & 4 \\
\hline 58 & DSJC125.1.col & $\mathrm{n}=125 ; \mathrm{m}=736$ & 5 & 0.09 & 5 & 5 \\
\hline 59 & DSJC125.5.col & $n=125 ; m=3891$ & - & 0.50 & 17 & 17 \\
\hline 60 & R125.1.col & $\mathrm{n}=125 ; \mathrm{m}=209$ & - & 0.03 & 5 & 5 \\
\hline 61 & R125.1c.col & $\mathrm{n}=125 ; \mathrm{m}=7501$ & - & 0.97 & 46 & 46 \\
\hline 62 & R125.5.col & $\mathrm{n}=125 ; \mathrm{m}=3838$ & - & 0.50 & 36 & 36 \\
\hline 63 & R250.1.col & $\mathrm{n}=250 ; \mathrm{m}=867$ & - & 0.03 & 8 & 8 \\
\hline 64 & R250.1c.col & $\mathrm{n}=250 ; \mathrm{m}=30227$ & - & 0.97 & 64 & 64 \\
\hline 65 & flat300_28_0.col & $\mathrm{n}=300 ; \mathrm{m}=21695$ & 28 & 0.48 & 31 & 28 \\
\hline
\end{tabular}

Table 4. Computation of Near Optimal Solution on Large Graphs $(\mathbf{n} \geq \mathbf{5 0 0})$

\begin{tabular}{|c|c|c|c|c|c|c|}
\hline \multirow{2}{*}{ Graph No } & \multicolumn{4}{|c|}{ Graph (G) } & \multicolumn{2}{|c|}{ Minimum color obtained in } \\
\hline & Graph Type & Instances & $\chi(\mathrm{G})$ & Density & Existing method & Proposed method \\
\hline 66 & latin_square_10.col & $\mathrm{n}=900 ; \mathrm{m}=307350$ & - & 0.76 & 97 & 97 \\
\hline 67 & homer.col & $\mathrm{n}=561 ; \mathrm{m}=1629$ & 13 & 0.01 & 13 & 13 \\
\hline 68 & inithx.i.1.col & $\mathrm{n}=864 ; \mathrm{m}=18707$ & 54 & 0.05 & 54 & 54 \\
\hline 69 & inithx.i.2.col & $\mathrm{n}=645 ; \mathrm{m}=13979$ & 31 & 0.07 & 31 & 31 \\
\hline 70 & inithx.i.3.col & $\mathrm{n}=621 ; \mathrm{m}=13969$ & 31 & 0.07 & 31 & 31 \\
\hline 71 & DSJC500.5.col & $\mathrm{n}=500 ; \mathrm{m}=62624$ & - & 0.50 & 48 & 48 \\
\hline 72 & DSJR500.1.col & $\mathrm{n}=500 ; \mathrm{m}=3555$ & - & 0.03 & 12 & 12 \\
\hline 73 & DSJR500.5.col & $\mathrm{n}=500 ; \mathrm{m}=58862$ & 122 & 0.47 & 122 & 122 \\
\hline 74 & DSJC1000.5.col & $\mathrm{n}=1000 ; \mathrm{m}=249826$ & - & 0.50 & 83 & 83 \\
\hline 75 & R1000.1.col & $\mathrm{n}=1000 ; \mathrm{m}=14378$ & - & 0.03 & 20 & 20 \\
\hline 76 & flat1000_50_0.col & $\mathrm{n}=1000 ; \mathrm{m}=245000$ & 50 & 0.49 & 50 & 50 \\
\hline 77 & flat1000_60_0.col & $\mathrm{n}=1000 ; \mathrm{m}=245830$ & 60 & 0.49 & 60 & 60 \\
\hline 78 & flat1000_76_0.col & $n=1000 ; m=246708$ & 76 & 0.49 & 82 & 80 \\
\hline
\end{tabular}

Table 5. Comparison of proposed method with well known and recent methods [28, 30-31, 51-52]

\begin{tabular}{|c|c|c|c|c|c|c|c|}
\hline \multirow[b]{2}{*}{ Graph } & \multirow[b]{2}{*}{$\chi(G)$} & \multicolumn{6}{|c|}{ Near optimal color obtained obtained using } \\
\hline & & TS & HCA & RLF & $\begin{array}{l}\text { Local search me- } \\
\text { thod }\end{array}$ & $\begin{array}{l}\text { Hybrid and population-based me- } \\
\text { thod }\end{array}$ & $\begin{array}{l}\text { Proposed me- } \\
\text { thod }\end{array}$ \\
\hline le450_25c.col & 25 & 26 & 26 & 27 & 25 & 25 & 25 \\
\hline le450_25d.col & 25 & 26 & 26 & 28 & 25 & 25 & 25 \\
\hline flat300_28.col & 28 & 32 & 31 & 37 & 28 & 29 & 28 \\
\hline flat1000_76_0.col & 76 & 87 & 83 & 106 & 85 & 82 & 80 \\
\hline latin_square_10.col & - & 130 & 127 & 122 & 99 & 97 & 97 \\
\hline
\end{tabular}




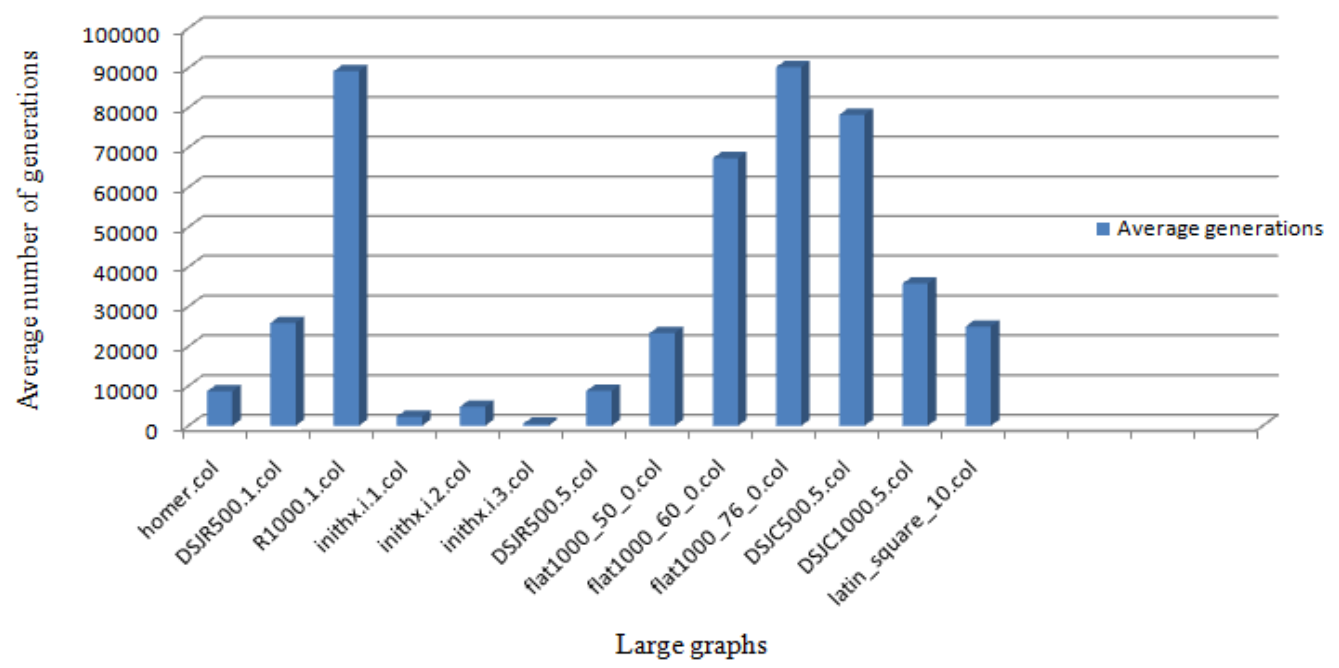

Figure 4. Expected generations obtained for large graphs.

Table 6. C(F) obtained for various benchmark instances

\begin{tabular}{cccc}
\hline Problem & Number of Cells & Number of Channels & C(F) \\
\hline Ex1 & 4 & 11 & 0 \\
Ex2 & 5 & 17 & 0 \\
HEX1 & 21 & 37 & 0 \\
HEX2 & 21 & 91 & 0 \\
HEX3 & 21 & 21 & 0 \\
HEX4 & 21 & 56 & 0 \\
KUNZ1 & 10 & 30 & 0 \\
KUNZ2 & 15 & 44 & 0 \\
KUNZ3 & 20 & 60 & 0 \\
KUNZ4 & 25 & 73 & 0 \\
\hline
\end{tabular}

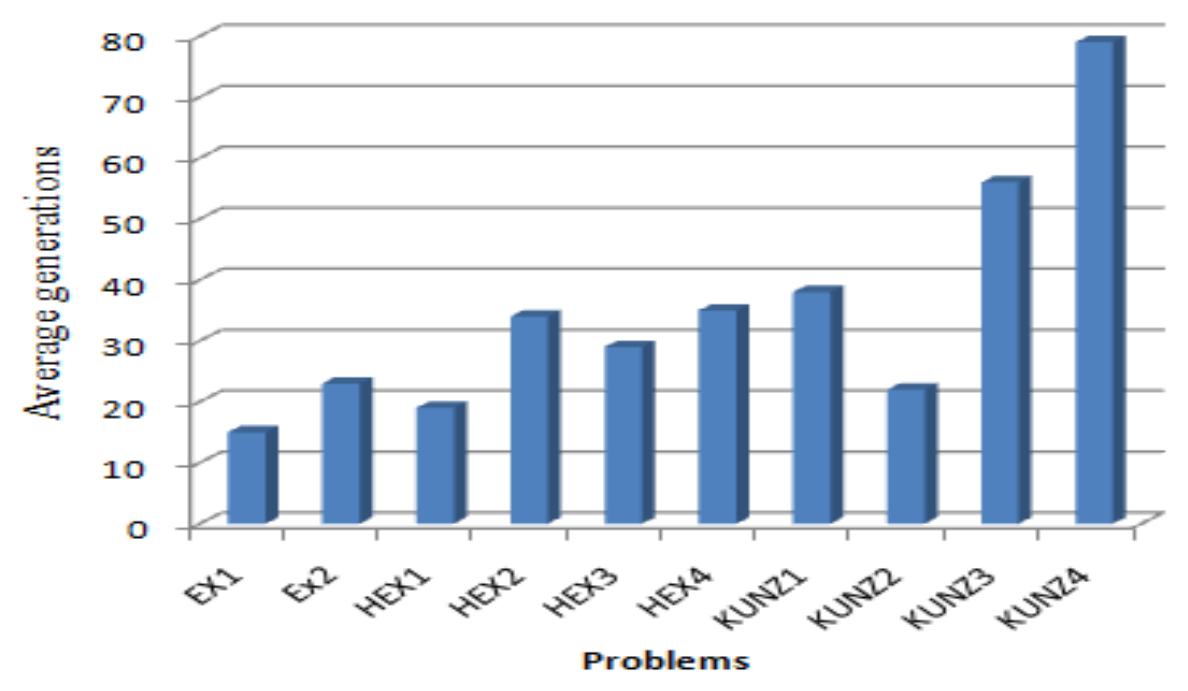

Figure 5. Expected generations obtained for CAP instances. 
Table 7. Frequency of Convergence

\begin{tabular}{ccccc}
\hline Problem & No. of Cells $(\mathrm{n})$ & No. of Channels $(\mathrm{m})$ & Simulation Run Length & Frequency of Convergence \\
\hline 1 & 4 & 11 & 100 & $100 \%$ \\
2 & 25 & 73 & 20000 & $100 \%$ \\
3 & 21 & 385 & 20000 & $100 \%$ \\
4 & 500 & 1250 & 40000 & $100 \%$ \\
5 & 1000 & 1500 & 45000 & $100 \%$ \\
6 & 2000 & 1750 & 50000 & $99.6 \%$ \\
7 & 5000 & 2000 & 50000 & $98.7 \%$ \\
8 & 10000 & 2500 & 50000 & $98.9 \%$ \\
10 & 20000 & 5000 & 50000 & $97.9 \%$ \\
\hline
\end{tabular}

\section{Conclusion}

A new multi-objective evolutionary algorithm using new crossover and mutation operators with multi-objectives in finding the solution to graph coloring and its applications are devised in this research work. The outcomes of the devised evolutionary method are compared with other strategies. The method of selecting multiple better gene sequences and applying them in subsequent operations with multiple objectives using the devised operators minimized the searching significantly and also reduces the average generations compared to the standard genetic algorithm. The proposed operators achieve better solution compared to other methods. The expected performance measures are also increased while minimizing the expected generations. The proposed operators can also be applied in solving special engineering applications of graph coloring. The clique split strategy reduce the interference of CAP with minimal $\mathrm{N}$ and hence better performance is obtained compared to the well known methods. Stochastic convergence of higher frequency of convergence is attained for the standard and random CAPs of large sizes.

The scope for future work includes the following:

- $\quad$ Devising statistical based evolutionary operators for further state space reduction

- Improving the performance of the devised operators with proper searching strategy to minimize the generations

- $\quad$ Enhancing the results by devising a parallel evolutionary method

- $\quad$ Designing the new genetic programming in solving applications of coloring

\section{References}

[1] Balakrishnan, R. and Ranganathan, K. (2000). A Textbook of Graph Theory. $1^{\text {st }}$ Edition, Springer-Verlag, New York Publisher.

[2] Michael R. Garey and David S. (1979). Johnson: Computers and Intractability: A Guide to the Theory of NP-Completeness. W. H. Freeman and Company, New York.

[3] Timir Maitra, Anindya J. Pal, Debnath Bhattacharyya, and Tai-hoon Kim. (2010). Noise Reduction in VLSI Circuits using Modified GA Based Graph Coloring. International Journal of Control and Automation, 3(2), (2010).

[4] Hertz, A. and Werra D. de. (1987). Using tabu search techniques for graph coloring. Computing, 39(4), 345-351.

[5] Charles Fleurent and Jacques A. Ferland. (1996). Genetic and hybrid algorithms for graph coloring. Annals of Operations Research, 63(3), 437-461.

[6] Christine, L. (2006). Mumford: New Order Based Crossovers for the Graph Coloring Problem. Parallel Problem Solving from Nature, 4193, 880-889.

[7] Anuj Mehrotra and Michael A. Trick. (1995). A Column Generation Approach for Graph Coloring. INFORMS Journal on Computing, 8, 344-354.

[8] Isabel Méndez-Díaz and Paula Zabala. A Branch and Cut algorithm for graph coloring. Discrete Applied Mathematics, 154, 826-847.

[9] Remi Monasson. (2004). On the Analysis of Backtrack Procedures for the Coloring of Random Graphs. Lect.Notes Phys., 650, 235-254. 
[10] Lixia Han and Zhanli Han. (2010). A Novel Bi-objective Genetic Algorithm for the Graph Coloring Problem. $2^{\text {nd }}$ International Conference on Computer Modeling and Simulation.

[11] Tamás Szép and Zoltán Ádám Mann. (2010). Graph coloring: The more colors, the better? $11^{\text {th }}$ International Symposium on Computational Intelligence and Informatics (CINTI).

[12] Rudolph and Günter. (1998). Finite Markov Chain Results in Evolutionary Computation: A Tour d'Horizon. Fundamenta Informaticae, 35(1-4), 67-89.

[13] A. E. Eiben, J. K. Van Der Hauw, and J. I. Van Hemert. (1998). Graph Coloring with Adaptive Evolutionary Algorithms. Journal of Heuristics, 4, 25-46.

[14] Philippe Galinier and Alain Hertz. (2006). A survey of local search methods for graph coloring. Computers \& Operations Research, 33(9), 2547-2562.

[15] David S. Johnson, Cecilia R. Aragon, Lyle A. McGeoch, and Catherine Schevon. (1991). An Experimental Evaluation; Part II, Graph Coloring and Number Partitioning. Operations Research, 39(3).

[16] David S. Johnson and Michael A. Trick. (1993). Cliques, Coloring, and Satisfiability. American Mathematical Society, 26.

[17] Kazunori Mizuno and Seiichi Nishihara. (2008). Constructive generation of very hard 3-colorability instances. Discrete Applied Mathematics, 156, 218-229.

[18] Blanca Cases, Carmen Hernandez, Manuel Grana, and Alicia D’Anjou. (2008). On the ability of Swarms to compute the 3-coloring of graphs. Artificial Life, (2008).

[19] Manuel Graña, Blanca Cases, Carmen Hernandez, and Alicia D’Anjou. (2010). Further Results on Swarms Solving Graph Coloring. ICCSA 2010: Computational Science and Its Applications, 541-551.

[20] Yongquan Zhou, Hongqing Zheng, Qifang Luo, and Jinzhao Wu. (2013). An improved Cuckoo Search Algorithm for Solving Planar Graph Coloring Problem. Applied Mathematics \& Information Sciences, 7(2), 785-792.

[21] Soma Saha, Rajeev Kumar, and Gyan Baboo. (2013). Characterization of graph properties for improved Pareto fronts using heuristics and EA for bi-objective graph coloring problem. Applied Soft Computing, 13(5), 2812-2822.

[22] Steven Prestwich. (2008). Generalised graph colouring by a hybrid of local search and constraint programming. Discrete Applied Mathematics, 156, 148-158.

[23] Igor Dukanovic and Franz Rendl. (2008). A semidefinite programming-based heuristic for graph coloring. Discrete Applied Mathematics, 156, 180-189.

[24] Thang N. Bui, ThanhVu H. Nguyen, Chirag M. Patel, and Kim-Anh T. Phan. (2008). An ant-based algorithm for coloring graphs. Discrete Applied Mathematics, 156, 190-200.

[25] Ling-Yuan Hsu, Shi-Jinn Horng, Pingzhi Fan, Muhammad Khurram Khan, Yuh-Rau Wang, Ray-Shine Run, Jui-Lin Lai, and Rong-Jian Chen. (2011). MTPSO algorithm for solving planar graph coloring problem. Expert Systems with Applications, 38, 5525-5531.

[26] Guangzhao Cui, Limin Qin, Sha Liu, Yanfeng Wang, Xuncai Zhang, and Xianghong Cao. (2008). Modified PSO algorithm for solving planar graph coloring problem. Progress in Natural Science, 18, 353-357.

[27] The Graph Coloring instances. http://mat.gsia.cmu.edu/COLOR/instances.html.

[28] Hugo Hernández and Christian Blum. (2014). FrogSim: Distributed graph coloring in wireless ad hoc networks. Telecommunication Systems, 55(2), 211-223.

[29] Junichi Yoshino and Isao Ohtomo. (2005). Study on efficient channel assignment method using the genetic algorithm for mobile communication systems. Soft Computing, 9(2), 143-148.

[30] Pablo San Segundo. (2012). A new DSATUR-based algorithm for exact vertex coloring. Computers \& Operations Research, 39(7), 1724-1733.

[31] Philippe Galinier and Jin-Kao Hao. (1999). Hybrid Evolutionary Algorithms for Graph Coloring. Journal of Combinatorial Optimization, 3(4), 379-397.

[32] Raja Marappan and Gopalakrishnan Sethumadhavan. (2013). A new genetic algorithm for graph coloring. $5^{\text {th }}$ International Conference on Computational Intelligence, Modelling and Simulation, 49-54, (2013).

[33] Gopalakrishnan Sethumadhavan and Raja Marappan. (2013). A Genetic Algorithm for Graph Coloring using Single Parent Conflict Gene Crossover and Mutation with Conflict Gene Removal Procedure. IEEE International Conference on Computational Intelligence and Computing Research, 350-355, (2013).

[34] Xiang'en Chen, Yue Zu, Jin Xu, Zhiwen Wang, and Bing Yao. (2011). Vertex-Distinguishing E-Total Colorings of Graphs. Arabian Journal for Science and Engineering, 36(8), 1485-1500, (2011).

[35] Lewis, R. M. R. (2016). A Guide to Graph Coloring, Algorithms and Applications. Springer International Publishing Switzerland (2016).

[36] Marc Demange, Tinaz Ekim, Bernard Ries, and Cerasela Tanasescu. (2014). On some applications of the selective graph coloring problem. European Journal of Operational Research, (2014). 
[37] Marc Demange, Tinaz Ekim, and Bernard Ries. (2015). On the minimum and maximum selective graph coloring problems in some graph classes. Discrete Applied Mathematics, (2015).

[38] Shih Heng Cheng and Ching Yao Huang. (2013). Coloring-Based Inter-WBAN Scheduling for Mobile Wireless Body Area Networks. IEEE Transactions on Parallel and Distributed Systems, 24(2), (2013).

[39] Elmahdi Driouch and Wessam Ajib. (2013). Downlink Scheduling and Resource Allocation for Cognitive Radio MIMO Networks. IEEE Transactions on Vehicular Technology, 62(8), (2013).

[40] Elmahdi Driouch and Wessam Ajib. (2012). Efficient Scheduling Algorithms for Multiantenna CDMA Systems. IEEE Transactions on Vehicular Technology, 61(2), (2012).

[41] Matti Peltomäki, Juha-Matti Koljonen, Olav Tirkkonen, and Mikko Alava. (2012). Algorithms for Self-Organized Resource Allocation in Wireless Networks. IEEE Transactions on Vehicular Technology, 61(1), (2012).

[42] Lynn Takeshita. (2016). Coloring Signed Graphs, (2016).

[43] Edita Macajov, Andre Raspaud, and Martin Skoviera. (2016). The chromatic number of a signed graph.

[44] Mohamed Abdelfattah and Ahmed Shawish. (2016). Automated Academic Schedule Builder for University's Faculties. Proceedings of the World Congress on Engineering, (2016).

[45] Sandeep Saharan and Ravinder Kumar. (2016). Graph Coloring based Optimized Algorithm for Resource Utilization in Examination Scheduling. Applied Mathematics \& Information Sciences, (2016).

[46] Ferdous M. O. Tawfiq and Kholoud Khalid S. Al-qahtani. (2016). Graph Coloring Applied to Medical Doctors Schedule. The $10^{\text {th }}$ International Conference on Advanced Engineering Computing and Applications in Sciences, (2016).

[47] Simon Thevenin, Nicolas Zufferey, and Jean-Yves Potvin. (2016). Graph multi-coloring for a job scheduling application. CIRRELT, (2016).

[48] Bing Zhou. (2016). On the Maximum Number of Dominating Classes in Graph Coloring. Open Journal of Discrete Mathematics, (2016).

[49] Serge Gaspers and Edward J. Lee. (2016). Faster Graph Coloring in Polynomial Space.

[50] Angelini, P., Bekos, M. A., De Luca, F., Didimo, W., Kaufmann, M., Kobourov, S., Montecchiani, F., Raftopoulou, C. N., Roselli, V., and Symvonis, A. (2017). Vertex-Coloring with Defects. Journal of Graph Algorithms and Applications, (2017).

[51] Murat Aslan and Nurdan Akhan Baykan. (2016). A Performance Comparison of Graph Coloring Algorithms. International Journal of Intelligent Systems and Applications in Engineering, (2016).

[52] Severino F. Galán. (2017). Simple decentralized graph coloring. Computational Optimization and Applications, (2017).

[53] T. Veeramakali, S. Jayashri, and S. Prabu. (2017). Intelligent dynamic spectrum allocation with bandwidth flexibility in cognitive radio network. Cluster Computing, 20: 1575-1586, (2017).

[54] I. Nelson, C. Annadurai, R. Kalidoss, and B. Partibane. (2017). Mitigation of co-channel interferences in cognitive multi-carrier code division multiple access system by singular value decomposition techniques. Cluster Computing. DOI: 10.1007/s10586-017-0997-y (2017).

[55] Xuesong Yan, Hanmin Liu, Zhixin Zhu, and Qinghua Wu. (2017). Hybrid genetic algorithm for engineering design problems. Cluster Computing, 20: 263-275.

[56] Xiangyu Meng, Liyan Dong, Yongli Li, and WilliamW. Guo. (2017). A genetic algorithm using K-path initialization for community detection in complex networks. Cluster Computing, 20: 311-320.

[57] Marappan, R. and Sethumadhavan, G. (2018). Solution to graph coloring using genetic and tabu search procedures. Arab J Sci Eng., 2018, 43, 525-542. Doi: https://doi.org/10.1007/s13369-017-2686-9.

[58] Marappan, R. and Sethumadhavan, G. (2020). Complexity analysis and stochastic convergence of some well-known evolutionary operators for solving graph coloring problem. Mathematics, 2020, 8, 303 . doi: https://doi.org/10.3390/math8030303. 